\title{
Comparison of European and American Athletes in Jumping Events in Terms of Competition Season Variables: Different Continents, Different Education of Coaching
}

\author{
Iş1k Bayraktar \\ Department of Coaching Education, Faculty of Sport Sciences \\ Alanya Alaaddin Keykubat University, Alanya, Turkey \\ Tel: 90-242-510-6150Ｅ-mail: isik.bayraktar@alanya.edu.tr \\ Tuncay Örs \\ Ministry of National Education, Aydin, Turkey \\ Tel: 90-256-544-1049_E-mail: tuncay400@gmail.com

\begin{abstract}
Berfin Serdil Örs (Corresponding author)
Department of Coaching Education, Faculty of Sport Sciences

Aydin Adnan Menderes University, Aydin, Turkey

Tel: 90-256-315-3538Ｅ-mail: bsutcu@adu.edu.tr
\end{abstract}

Received: August 1, 2020 Accepted: August 29, 2020 Published: September 7, 2020

doi:10.5296/jei.v6i2.17468 URL: https://doi.org/10.5296/jei.v6i2.17468

\begin{abstract}
It is predicted that athletes can achieve high efficiency levels when training contents are applied within the scope of annual plans prepared on a scientific basis. Annual plans consist of preparation, competition and interim periods. Topics such as determining target competition where the peak performance will be revealed during competition period are examined under periodization title of training science. This study was carried out to determine the variables of the competition seasons of American and European athletes in
\end{abstract}


jumping events and to identify the similarities and differences between the variables. The study group consisted of elite American and European athletes, who had ranked in the top 100 in the world charts of the 2018 season. The differences between athletes were determined by Mann Whitney U-Test. In terms of the number of competition season days, number of days between competitions and final performance value of season, statistically significant differences were found between the athletes in favor of the American male long jumpers and male triple jumpers $(\mathrm{p}<0.05)$. Significant differences were determined between the European and American high jumpers in terms of number of days between competitions in both genders $(p<0.05)$. In the pole vault event, the total number of competitions participated by both male and female European athletes were higher than total number of competitions participated by American athletes $(p<0.05)$. As a result, it was thought that the differences determined between the groups such as the number of days between competitions may be due to the differences in the perception of periodization and different coaching educations embraced by continents.

Keywords: coaching education, periodization, competitive phase, athletics, season best, prediction equation

\section{Introduction}

Athletics, first emerged in the 19th century in colleges and universities in Great Britain. The athletics rules that are applied all over the world today were determined by the International Amateur Athletics Federation (IAAF), which was established in Stockholm in 1912 and has more than 150 member countries (Demir, 2008). Olympic athletics events that are managed by the IAAF are divided into two as track and field competitions. Track competitions consist of runs, while field competitions consist of throwing events and jumping events. In addition, jumping events are separately grouped as horizontal jumps, namely long jumps and triple jumps, and vertical jumps, namely high jumps and pole vaults (IAAF, 2017). In long and triple jumps, athletes are expected to jump horizontally to the maximum distance they can reach, while in high jumps and pole vaults athletes are expected to achieve maximum performance on a vertical track. Although jumping events have similar characteristics, they differ from each other in terms of technical content and training. These four jumping events can be ordered from most simple to most complex as follows: long jump, triple jump, high jump and pole vault (Müller \& Ritzdorf, 2009).

Athletic performance can be described as carrying out a specific physical routine or procedures (Ekechukwu \& Isiguzo, 2019). On the other hand, performance planning is the process by which an athlete or a team aligns all available resources, including money, people, knowledge and time, to maximize the probability of achieving the best performance at the right time (Dick, 2007). The methodology to evaluate 'performance' among elite athletes has not yet been settled in the scientific literature (Raysmith et al., 2019). However, trainers can apply specific routines that aim to target the aspects of a training routine that their athlete requires such as speed, agility, stamina, conditioning and coordination, as they are able to accurately monitor the athlete's development and performance (Örs et al., 2019a). Scientists of the field of training science agree that, in the jumping events of athletics, just like in all 
sport events, if the aim, content, equipment and applied methods of training are determined according to annual plans prepared with regard to the goals high efficiency levels can be achieved. A typical training program for elite athletes is composed of three phases: (1) preparation, (2) competition and (3) transition. Each of these phases includes four specific types of preparation: (1) physical, (2) technical, (3) tactical and (4) psychological (Lidor et al., 2007). Additionally, the physical/psychological adaptations appropriate for the phases should be completed (Bompa, 1994).

Annual plans are centered around the competition period. The main aim of the competition phase is to utilize all training factors to improve the motor and psychological abilities of the athletes in order for them to achieve peak performance (Bompa, 1999). The achievement of successful performance in training and competitions is based on long-time planning (Blumenstein \& Orbach, 2020).

Topics such as determining the target competition where the peak performance will be revealed, the number of competitions before the peak performance, the total number of competitions in the season and the ordering of the competitions according to priority are examined under the periodization title of the field of training science (Açıkada, 2018). In other words, the concept of periodization is one of the fundamental subjects of training science, especially as it is considered to be the key to high-level performance in target competitions. The structure of training plans is likely to be dependent on a number of factors including the type of the sport and level of the athlete (Bradbury et al., 2020). In many sport events a year is divided into three main training phases namely preparation, competition and transition (Bompa, 1994; Matveyev, 1981). The competition order determined in the schedule according to the level and goals of the athlete is important for the design of the competition period and for the athlete to achieve peak performance in the target competition. First priority competitions are those from which peak form is expected (2-4 competitions), while second priority competitions are those from which high form is expected and are considered as preparatory for the ones in which peak form is expected (6-8 competitions). Third priority competitions, on the other hand, those that are considered to be training preparation for first and second priority competitions (8-10 competitions) (Açıkada \& Bayraktar, 2018).

Achievements and systematic approaches to sports led to the emergence of notions such as periodization and changed the approach to sports and performance, thus influencing the start of a new period. In the 1960s and 1970s scientists, especially those from Eastern Bloc countries, developed the concept of periodization (Matveyev, 1981). The adoption of notions such as periodization in western countries did not take place until the 1980s. In fact, in some countries, the adaption of such notions extended until the 1990s (Açıkada, 2018). Bompa (1999) adapted and popularized the concept of periodization in the West in the 1990s (Blumenstein \& Orbach, 2020), while in others parts of the world it was not shaped until the 2010s (Aç1kada, 2018). These developments were adopted very late in many western countries. Even today, several countries do not consider training science as a new discipline of science, and have not yet established the necessary systematic approach (Açıkada, 2018). The United States is one of these countries that has avoided this structure. In Europe, in the 1950 s, the science of education, which first emerged with a structure that aimed for the 


\section{Macrothink}

superiority of the socialist regime as in the Soviet Union and then East Germany, started to take shape with a different philosophy and principle (Açıkada, 2018). In light of these different understandings, the aim of the present study was to determine the variables of the competition seasons of American and European athletes in jumping events, and to identify the similarities and differences between these variables.

\section{Method}

\subsection{Participants}

The study group was composed of American and European male and female elite athletes who ranked in the top 100 in the world charts in the 2018 season.

\subsection{Research Design}

The competition information of the athletes in the season was obtained from the 2018 world rankings (WorldAthletics, 2009a) on the official website of the IAAF. Each athletes' date of birth, the dates of the competitions held during the season and the results the athletes achieved were recorded from the database. This study complied with the ethical principles stated by the Declaration of Helsinki.

The age of the athletes, the total number of days in the season, the number of days between the competitions, the total number of competitions, the number of competitions in which the season's best (SB) performance was achieved, the ratio of the competition in which the season's best performance was achieved to the total number of competitions (SB/Competition) the percentages of the first and end performances calculated according to the season's best performance were determined as the research variables.

\subsection{Statistical Analysis}

The normality of the quantitative data was analyzed by the Kolmogorov Smirnov test. Mann-Whitney $U$ test was performed for non-normally distributed data to identify the similarities and differences. IBM-SPSS 20.0 software was used for statistical analysis and the significancy level was determined as $\mathrm{p}<0.05$.

\section{Results}

The comparisons of the European and American athletes, in terms of gender with respect to the variables examined within the scope of the research are given in Tables 1-8. 
Table 1. Mann Whitney U-test results regarding the differences between the age and seasonal performance variables of the American and European male long jumpers

\begin{tabular}{|c|c|c|c|c|c|c|c|}
\hline Long Jump (Male) & Continent & $\mathbf{n}$ & $\begin{array}{l}\text { Median } \\
(25-75 \%)\end{array}$ & Mean Rank & Total Rank & $\mathbf{U}$ & $\mathbf{p}$ \\
\hline \multirow{2}{*}{ World Ranking } & America & 39 & $49(26-82)$ & 32.9 & 1284.0 & \multirow{2}{*}{448.0} & \multirow{2}{*}{0.12} \\
\hline & Europe & 33 & $66(36-92)$ & 40.7 & 1344.0 & & \\
\hline \multirow{2}{*}{ Age (years) } & America & 39 & $23(21-26)$ & 31.6 & 1230.5 & \multirow{2}{*}{386.0} & \multirow{2}{*}{$0.02 *$} \\
\hline & Europe & 33 & $25(23-28.5)$ & 42.4 & 1397.5 & & \\
\hline \multirow{2}{*}{$\begin{array}{l}\text { The number of competition } \\
\text { days in the season }\end{array}$} & America & 39 & $127(75-177)$ & 42.4 & 1653.5 & \multirow{2}{*}{406.5} & \multirow{2}{*}{$0.04 *$} \\
\hline & Europe & 33 & $97(75-120)$ & 29.5 & 974.5 & & \\
\hline \multirow{2}{*}{$\begin{array}{l}\text { The number of days } \\
\text { among competitions }\end{array}$} & America & 39 & $12(9-17)$ & 46.1 & 1798.0 & \multirow{2}{*}{360.0} & \multirow{2}{*}{$0.01 *$} \\
\hline & Europe & 33 & $9(7-11)$ & 25.2 & 830.0 & & \\
\hline \multirow{2}{*}{ The total number of competitions } & America & 39 & $9(7-12)$ & 34.1 & 1331.5 & \multirow{2}{*}{537.5} & \multirow{2}{*}{0.66} \\
\hline & Europe & 33 & $10(8-15)$ & 39.3 & 1296.5 & & \\
\hline \multirow{2}{*}{$\begin{array}{l}\text { The number of competitions in which } \\
\text { SB performance was achieved }\end{array}$} & America & 39 & $5(3-8)$ & 41.1 & 1604.5 & \multirow{2}{*}{559.0} & \multirow{2}{*}{0.86} \\
\hline & Europe & 33 & $5(2-10)$ & 31.0 & 1023.5 & & \\
\hline \multirow{2}{*}{ SB/Competition } & America & 39 & $62(40-82)$ & 38.1 & 1485.0 & \multirow{2}{*}{503.5} & \multirow{2}{*}{0.39} \\
\hline & Europe & 33 & $57(31-75)$ & 34.6 & 1143.0 & & \\
\hline \multirow{2}{*}{$\mathrm{SB}(\mathrm{m})$} & America & 39 & $8.08(7.98-8.24)$ & 34.9 & 1360.5 & \multirow{2}{*}{466.0} & \multirow{2}{*}{0.19} \\
\hline & Europe & 33 & $8.00(7.93-8.13)$ & 38.4 & 1267.5 & & \\
\hline \multirow{2}{*}{ First performance in a season $(\mathrm{m})$} & America & 39 & $7.64(7.50-7.92)$ & 35.5 & 1385.0 & \multirow{2}{*}{497.0} & \multirow{2}{*}{0.35} \\
\hline & Europe & 33 & $7.76(7.59-7.90)$ & 37.7 & 1243.0 & & \\
\hline \multirow{2}{*}{ Last performance in a season $(\mathrm{m})$} & America & 39 & $7.83(7.59-7.93)$ & 41.2 & 1605.5 & \multirow{2}{*}{408.5} & \multirow{2}{*}{$0.04 *$} \\
\hline & Europe & 33 & $7.71(7.45-7.82)$ & 31.0 & 1022.5 & & \\
\hline \multirow{2}{*}{ Season performance average $(\mathrm{m})$} & America & 39 & $7.80(7.66-7.87)$ & 38.8 & 1511.5 & & \\
\hline & Europe & 33 & $7.73(7.66-7.84)$ & 33.8 & 1116.5 & 491.5 & 0.31 \\
\hline & America & 39 & $95(92-97)$ & 31.9 & 1244.5 & & \\
\hline rirst competilton \% & Europe & 33 & $96(94-98.5)$ & 41.9 & 1383.5 & & 0.68 \\
\hline & America & 39 & $96(94-98)$ & 38.2 & 1489.5 & & \\
\hline Last competition \% & Europe & 33 & $96(93-98)$ & 34.5 & 1138.5 & 492.5 & 0.31 \\
\hline & America & 39 & $96(95-97)$ & 32.7 & 1273.5 & & \\
\hline Average $\%$ & Europe & 33 & $97(95.5-97)$ & 41.1 & 1354.5 & 503.0 & 0.36 \\
\hline
\end{tabular}

Note. ${ }^{*} \mathrm{p}<0.05$.

When the male athletes in the horizontal jumping events were compared, it was observed that the European athletes had higher age values. In terms of the number of competition season days, the number of days among competitions and the last performance of the season, statistically significant differences were determined between the athletes in favor of the 
American male long jumpers (Table 1) $(\mathrm{p}<0.05)$.

Table 2. Comparison of the averages of the age and seasonal performance variables of the American and European female long jumpers

\begin{tabular}{|c|c|c|c|c|c|c|c|}
\hline Long Jump (Female) & Continent & $\mathbf{n}$ & $\begin{array}{l}\text { Median } \\
(25-75 \%)\end{array}$ & Mean Rank & Total Rank & $\mathbf{U}$ & $\mathbf{p}$ \\
\hline \multirow{2}{*}{ World Ranking } & America & 37 & $61(31-93)$ & 48.5 & 1792.5 & \multirow{2}{*}{760.5} & \multirow{2}{*}{0.16} \\
\hline & Europe & 50 & $47(24-82)$ & 40.7 & 2035.5 & & \\
\hline \multirow{2}{*}{ Age (years) } & America & 37 & $25(21.5-27.5)$ & 38.9 & 1439.5 & \multirow{2}{*}{736.5} & \multirow{2}{*}{0.11} \\
\hline & Europe & 50 & $27(23-30)$ & 47.8 & 2388.5 & & \\
\hline \multirow{2}{*}{$\begin{array}{l}\text { The number of competition } \\
\text { days in the season }\end{array}$} & America & 37 & $100(85-152)$ & 48.9 & 1810.5 & \multirow{2}{*}{742.5} & \multirow{2}{*}{0.12} \\
\hline & Europe & 50 & $96(75-121)$ & 40.4 & 2017.5 & & \\
\hline \multirow{2}{*}{ The number of days among competitions } & America & 37 & $12(9-18)$ & 50.6 & 1873.5 & \multirow{2}{*}{679.5} & \multirow{2}{*}{$0.03 *$} \\
\hline & Europe & 50 & $10(9-13)$ & 39.1 & 1954.5 & & \\
\hline \multirow{2}{*}{ The total number of competitions } & America & 37 & $9(6-11)$ & 42.8 & 1583.0 & \multirow{2}{*}{880.0} & \multirow{2}{*}{0.70} \\
\hline & Europe & 50 & $9(7-12)$ & 44.9 & 2245.0 & & \\
\hline \multirow{2}{*}{$\begin{array}{l}\text { The number of competitions } \\
\text { in which SB was achieved }\end{array}$} & America & 37 & $4(3-7)$ & 44.0 & 1627.5 & \multirow{2}{*}{924.5} & \multirow{2}{*}{1.00} \\
\hline & Europe & 50 & $5(2-7)$ & 44.0 & 2200.5 & & \\
\hline \multirow{2}{*}{ SB/Competition } & America & 37 & $50(33-79)$ & 44.8 & 1656.0 & \multirow{2}{*}{897.0} & \multirow{2}{*}{0.81} \\
\hline & Europe & 50 & $56.5(33-68.8)$ & 43.4 & 2172.0 & & \\
\hline \multirow{2}{*}{$\mathrm{SB}(\mathrm{m})$} & America & 37 & $6.60(6.50-6.70)$ & 40.5 & 1500.0 & \multirow{2}{*}{797.0} & \multirow{2}{*}{0.27} \\
\hline & Europe & 50 & $6.65(6.53-6.72)$ & 46.6 & 2328.0 & & \\
\hline \multirow{2}{*}{ First performance in the season (m) } & America & 37 & $6.31(6.12-6.53)$ & 41.4 & 1531.0 & \multirow{2}{*}{828.0} & \multirow{2}{*}{0.41} \\
\hline & Europe & 50 & $6.38(6.17-6.52)$ & 45.9 & 2297.0 & & \\
\hline \multirow{2}{*}{ Last performance in the season (m) } & America & 37 & $6.22(6.05-6.37)$ & 33.5 & 1239.0 & \multirow{2}{*}{536.0} & \multirow{2}{*}{$0.001 *$} \\
\hline & Europe & 50 & $6.40(6.21-6.53)$ & 51.8 & 2589.0 & & \\
\hline \multirow{2}{*}{ Season performance average $(\mathrm{m})$} & America & 37 & $6.31(6.22-6.44)$ & 36.2 & 1340.0 & & \\
\hline & Europe & 50 & $6.40(6.3-6.53)$ & 49.8 & 2488.0 & 001.0 & 0.01 \\
\hline & America & 37 & $96(94-97.5)$ & 43.4 & 1607.0 & & \\
\hline rirst competilion \% & Europe & 50 & $95.5(94-98)$ & 44.4 & 2221.0 & 904.0 & 0.00 \\
\hline tition 0 & America & 37 & $94(92-96)$ & 34.1 & 1261.5 & 5505 & $0002 *$ \\
\hline Lase Competilivil & Europe & 50 & $96(94-98)$ & 51.3 & 2566.5 & 30.0 & 0.002 \\
\hline & America & 37 & $96(95-97)$ & 37.7 & 1395.0 & & \\
\hline & Europe & 50 & $97(95-97.3)$ & 48.7 & 2433.0 & - & \\
\hline
\end{tabular}

$* \mathrm{p}<0.05$. 
When the female long jump athletes were compared in terms of the competition variables (Table 2) it was found that there were statistically significant differences in favor of the American athletes in terms of the number of days between competitions, final season performance, season average performance and SB/competition variables in favor of the European athletes $(\mathrm{p}<0.05)$.

Table 3. Comparison of the averages of the age and seasonal performance variables of the American and European male triple jumpers

\begin{tabular}{|c|c|c|c|c|c|c|c|}
\hline Triple Jump (Male) & Continent & $\mathbf{n}$ & Median (25-75\%) & Mean Rank & Total Rank & $\mathbf{U}$ & $\mathbf{p}$ \\
\hline \multirow{2}{*}{ World Ranking } & America & 31 & $34(9-77)$ & 30.5 & 944.0 & \multirow{2}{*}{448.0} & \multirow{2}{*}{0.12} \\
\hline & Europe & 37 & $52(33-73)$ & 37.9 & 1402.0 & & \\
\hline \multirow{2}{*}{ Age (years) } & America & 31 & $22(21-26)$ & 28.5 & 882.0 & \multirow{2}{*}{386.0} & \multirow{2}{*}{$0.02 *$} \\
\hline & Europe & 37 & $25(22-29.5)$ & 39.6 & 1464.0 & & \\
\hline \multirow{2}{*}{$\begin{array}{l}\text { The number of competition } \\
\text { days in the season }\end{array}$} & America & 31 & $117(76-139)$ & 39.9 & 1236.5 & \multirow{2}{*}{406.5} & \multirow{2}{*}{$0.04 *$} \\
\hline & Europe & 37 & $91(76-113)$ & 30.0 & 1109.5 & & \\
\hline \multirow{2}{*}{ The number of days among competitions } & America & 31 & $15(12-17)$ & 41.4 & 1283.0 & \multirow{2}{*}{360.0} & \multirow{2}{*}{$0.01 *$} \\
\hline & Europe & 37 & $12(9-13)$ & 28.7 & 1063.0 & & \\
\hline \multirow{2}{*}{ The total number of competitions } & America & 31 & $8(6-10)$ & 33.3 & 1033.5 & \multirow{2}{*}{537.5} & \multirow{2}{*}{0.66} \\
\hline & Europe & 37 & $9(7-10)$ & 35.5 & 1312.5 & & \\
\hline \multirow{2}{*}{$\begin{array}{l}\text { The number of competitions in } \\
\text { which SB was achieved }\end{array}$} & America & 31 & $5(3-7)$ & 35.0 & 1084.0 & \multirow{2}{*}{559.0} & \multirow{2}{*}{0.86} \\
\hline & Europe & 37 & $5(2-7)$ & 34.1 & 1262.0 & & \\
\hline \multirow{2}{*}{ SB/Competition } & America & 31 & $57(43-88)$ & 36.8 & 1139.5 & \multirow{2}{*}{503.5} & \multirow{2}{*}{0.39} \\
\hline & Europe & 37 & $57(33-77.5)$ & 32.6 & 1206.5 & & \\
\hline \multirow{2}{*}{ SB Performance (m) } & America & 31 & $16.83(16.59-17.34)$ & 38.0 & 1177.0 & \multirow{2}{*}{466.0} & \multirow{2}{*}{0.19} \\
\hline & Europe & 37 & $16.74(16.61-16.88)$ & 31.6 & 1169.0 & & \\
\hline \multirow{2}{*}{ First performance in the season $(\mathrm{m})$} & America & 31 & $16.40(16.03-17.02)$ & 37.0 & 1146.0 & \multirow{2}{*}{497.0} & \multirow{2}{*}{0.35} \\
\hline & Europe & 37 & $16.39(15.94-16.66)$ & 32.4 & 1200.0 & & \\
\hline \multirow{2}{*}{ Last performance in the season $(\mathrm{m}$} & America & 31 & $16.53(16.05-16.96)$ & 39.8 & 1234.5 & \multirow{2}{*}{408.5} & \multirow{2}{*}{$0.04 *$} \\
\hline & Europe & 37 & $16.31(15.96-16.58)$ & 30.0 & 1111.5 & & \\
\hline \multirow{2}{*}{ Season performance average (m) } & America & 31 & $16.28(16.15-16.97)$ & 37.2 & 1151.5 & & \\
\hline & Europe & 37 & $16.33(16.11-16.45)$ & 32.3 & 1194.5 & 491.5 & 0.31 \\
\hline & America & 31 & 97 (94-99) & 33.4 & 1036.0 & & \\
\hline First competition \% & Europe & 37 & $97(95-100)$ & 35.4 & 1310.0 & 540.0 & 0.68 \\
\hline$I_{0}$ & America & 31 & $98(96-100)$ & 37.1 & 1150.5 & 100 & D 0 \\
\hline Last competition \% & Europe & 37 & 97 (95.5-99) & 32.3 & 1195.5 & 492.5 & 0.31 \\
\hline & America & 31 & 97 (97-98) & 36.8 & 1140.0 & & \\
\hline Average \% & Europe & 37 & 97 (96-98) & 32.6 & 1206.0 & 503.0 & 0.36 \\
\hline
\end{tabular}

Note. ${ }^{*} \mathrm{p}<0.05$. 
In terms of the number of competition season days, the number of days among competitions and the last performance of the season, statistically significant differences were determined between the athletes in favor of the American triple jumpers (Table 3$)(p<0.05)$.

Table 4. Comparison of the averages of the age and seasonal performance variables of the American and European female triple jumpers

\begin{tabular}{|c|c|c|c|c|c|c|c|}
\hline Triple Jump (Female) & Continent & n & Median (25-75\%) & Mean Rank & Total Rank & $\mathbf{U}$ & $\mathbf{p}$ \\
\hline \multirow{2}{*}{ World Ranking } & America & 25 & $53(9-83)$ & 36.6 & 916.0 & \multirow{2}{*}{591.0} & \multirow{2}{*}{0.44} \\
\hline & Europe & 53 & $46(27-82)$ & 40.9 & 2165.0 & & \\
\hline \multirow{2}{*}{ Age (years) } & America & 25 & $26(22-28.5)$ & 41.2 & 1029.5 & \multirow{2}{*}{620.5} & \multirow{2}{*}{0.65} \\
\hline & Europe & 53 & $25(22-28)$ & 38.7 & 2051.5 & & \\
\hline \multirow{2}{*}{$\begin{array}{l}\text { The number of competition } \\
\text { days in the season }\end{array}$} & America & 25 & $118(84-157)$ & 51.7 & 1292.0 & \multirow{2}{*}{358.0} & \multirow{2}{*}{$0.001 *$} \\
\hline & Europe & 53 & $88(69-109)$ & 33.8 & 1789.0 & & \\
\hline \multirow{2}{*}{$\begin{array}{l}\text { The number of days } \\
\text { among competitions }\end{array}$} & America & 25 & $16(12-20)$ & 57.2 & 1431.0 & \multirow{2}{*}{219.0} & \multirow{2}{*}{$0.000 *$} \\
\hline & Europe & 53 & $10(9-12)$ & 31.1 & 1650.0 & & \\
\hline \multirow{2}{*}{ The total number of competitions } & America & 25 & $7(6-9)$ & 31.7 & 793.5 & \multirow{2}{*}{468.5} & \multirow{2}{*}{$0.04 *$} \\
\hline & Europe & 53 & $9(7-10)$ & 43.2 & 2287.5 & & \\
\hline \multirow{2}{*}{$\begin{array}{l}\text { The number of competitions } \\
\text { in which SB was achieved }\end{array}$} & America & 25 & $3(2-6)$ & 31.7 & 792.5 & \multirow{2}{*}{467.5} & \multirow{2}{*}{$0.04 *$} \\
\hline & Europe & 53 & $5(4-7)$ & 43.2 & 2288.5 & & \\
\hline \multirow{2}{*}{ SB/Competition } & America & 25 & $50(36.5-71)$ & 34.5 & 862.5 & \multirow{2}{*}{537.5} & \multirow{2}{*}{0.18} \\
\hline & Europe & 53 & $67(44.5-83)$ & 41.9 & 2218.5 & & \\
\hline \multirow[b]{2}{*}{ SB Performance (m) } & America & 25 & $13.90(13.65-14.54)$ & 42.0 & 1049.5 & \multirow[b]{2}{*}{600.5} & \multirow[b]{2}{*}{0.51} \\
\hline & Europe & 53 & $13.93(13.66-14.18)$ & 38.3 & 2031.5 & & \\
\hline \multirow{2}{*}{ First performance in the season $(\mathrm{m})$} & America & 25 & $13.47(12.96-14.01)$ & 40.0 & 1001.0 & \multirow{2}{*}{649.0} & \multirow{2}{*}{0.89} \\
\hline & Europe & 53 & $13.51(13.10-13.74)$ & 39.3 & 2080.0 & & \\
\hline \multirow{2}{*}{ Last performance in the season $(\mathrm{m})$} & America & 25 & $13.63(13.28-14.14)$ & 43.5 & 1088.0 & \multirow{2}{*}{562.0} & \multirow{2}{*}{0.28} \\
\hline & Europe & 53 & $13.52(13.31-13.87)$ & 37.6 & 1993.0 & & \\
\hline \multirow{2}{*}{ Season performance average (m) } & America & 25 & $13.48(13.28-14.16)$ & 40.8 & 1020.5 & & \\
\hline & Europe & 53 & $13.63(13.27-13.81)$ & 38.9 & 2060.5 & 629.5 & 0.72 \\
\hline it: & America & 25 & $97(94-98.5)$ & 38.5 & 961.5 & L 1 & P 70 \\
\hline First competition \% & Europe & 53 & 97 (94-99) & 40.0 & 2119.5 & 636.5 & 0.78 \\
\hline$T$ & America & 25 & $98(96-99)$ & 42.6 & 1066.0 & -004 & | \\
\hline Last competition \% & Europe & 53 & 97 (96-99) & 38.0 & 2015.0 & 584.0 & 0.39 \\
\hline & America & 25 & $98(97-98)$ & 39.6 & 990.5 & & \\
\hline A & Europe & 53 & $98(97-98)$ & 39.4 & 2090.5 & 659.5 & 0.97 \\
\hline
\end{tabular}

Note. ${ }^{*} \mathrm{p}<0.05$. 
Among the female triple jump athletes (Table 4), the number of days of the competition season and the number of days among competitions were determined to be higher in the American athletes. In the comparisons made in terms of the total number of competitions and the competition in which competition SB performance was achieved, significant differences were found between the groups in favor of the European athletes $(p<0.05)$.

Table 5. Comparison of the averages of the age and seasonal performance variables of the American and European male high jumpers

\begin{tabular}{|c|c|c|c|c|c|c|c|}
\hline High Jump (Male) & Continent & $\mathbf{n}$ & Median (25-75\%) & Mean Rank & Total Rank & $\mathbf{U}$ & $\mathbf{p}$ \\
\hline \multirow{2}{*}{ World Ranking } & America & 33 & $60(27-109)$ & 39.5 & 1302.0 & \multirow{2}{*}{579.0} & \multirow{2}{*}{0.37} \\
\hline & Europe & 40 & $43(36-86)$ & 35.0 & 1399.0 & & \\
\hline \multirow{2}{*}{ Age (years) } & America & 33 & $23(20-26)$ & 34.1 & 1126.0 & \multirow{2}{*}{565.0} & \multirow{2}{*}{0.29} \\
\hline & Europe & 40 & $24.5(21.3-26)$ & 39.4 & 1575.0 & & \\
\hline \multirow{2}{*}{$\begin{array}{l}\text { The number of competition } \\
\text { days in the season }\end{array}$} & America & 33 & $102(78-144)$ & 41.4 & 1365.5 & \multirow{2}{*}{515.5} & \multirow{2}{*}{0.11} \\
\hline & Europe & 40 & $94(72-118)$ & 33.4 & 1335.5 & & \\
\hline \multirow{2}{*}{ The number of days among competitions } & America & 33 & $13(11-16)$ & 45.8 & 1512.5 & \multirow{2}{*}{368.5} & \multirow{2}{*}{$0.001 *$} \\
\hline & Europe & 40 & $10(9-13)$ & 29.7 & 1188.5 & & \\
\hline \multirow{2}{*}{ The total number of competitions } & America & 33 & $8(6-10)$ & 34.6 & 1141.0 & \multirow{2}{*}{580.0} & \multirow{2}{*}{0.37} \\
\hline & Europe & 40 & $8(6-12)$ & 39.0 & 1560.0 & & \\
\hline \multirow{2}{*}{$\begin{array}{l}\text { The number of competitions } \\
\text { in which SB was achieved }\end{array}$} & America & 33 & $4(2-6)$ & 35.8 & 1182.0 & \multirow{2}{*}{621.0} & \multirow{2}{*}{0.66} \\
\hline & Europe & 40 & $4(2-8)$ & 38.0 & 1519.0 & & \\
\hline \multirow{2}{*}{ SB/Competition } & America & 33 & $50(35.5-75.5)$ & 36.9 & 1218.0 & \multirow{2}{*}{657.0} & \multirow{2}{*}{0.97} \\
\hline & Europe & 40 & $52(29-79.8)$ & 37.1 & 1483.0 & & \\
\hline \multirow{2}{*}{ SB Performance (m) } & America & 33 & $2.25(2.22-2.28)$ & 34.6 & 1140.0 & \multirow{2}{*}{579.0} & \multirow{2}{*}{0.37} \\
\hline & Europe & 40 & $2.26(2.23-2.27)$ & 39.0 & 1561.0 & & \\
\hline \multirow{2}{*}{ First performance in the season (m) } & America & 33 & $2.20(2.15-2.23)$ & 34.2 & 1128.5 & \multirow{2}{*}{567.5} & \multirow{2}{*}{0.30} \\
\hline & Europe & 40 & $2.21(2.16-2.24)$ & 39.3 & 1572.5 & & \\
\hline \multirow{2}{*}{ Last performance in the season $(\mathrm{m})$} & America & 33 & $2.19(2.14-2.24)$ & 36.0 & 1189.0 & \multirow{2}{*}{628.0} & \multirow{2}{*}{0.72} \\
\hline & Europe & 40 & $2.20(2.15-2.24)$ & 37.8 & 1512.0 & & \\
\hline \multirow{2}{*}{ Season performance average $(\mathrm{m})$} & America & 33 & $2.19(2.17-2.22)$ & 35.4 & 1169.0 & & \\
\hline & Europe & 40 & $2.20(2.16-2.22)$ & 38.3 & 1532.0 & 608.0 & 0.56 \\
\hline & America & 33 & 97 (95-99) & 37.3 & 1230.5 & & \\
\hline First competition \% & Europe & 40 & 97 (95-98.8) & 36.8 & 1470.5 & 650.5 & 0.92 \\
\hline I oct & America & 33 & 97 (95-99) & 37.0 & 1222.0 & 6500 & 000 \\
\hline Last competition \% & Europe & 40 & 97 (95-99) & 37.0 & 1479.0 & 059.0 & 0.99 \\
\hline & America & 33 & 97 (96-98) & 36.7 & 1212.0 & 0 & \\
\hline Aver & Europe & 40 & 97 (96.3-98) & 37.2 & 1489.0 & 651.0 & 0.92 \\
\hline
\end{tabular}

Note. ${ }^{*} \mathrm{p}<0.05$. 
Statistically significant differences were found between the European and American high jumpers (male) in terms of the number of days between competitions $(p<0.05)$. It was observed that the American athletes had longer competition gaps (Tables 5).

Table 6. Comparison of the averages of the age and seasonal performance variables of the American and European female high jumpers

\begin{tabular}{|c|c|c|c|c|c|c|c|}
\hline High Jump (Female) & Continent & $\mathbf{n}$ & Median (25-75\%) & Mean Rank & Total Rank & $\mathbf{U}$ & $\mathbf{p}$ \\
\hline \multirow{2}{*}{ World Ranking } & America & 26 & $87(41-108)$ & 55.1 & 1433.5 & \multirow{2}{*}{555.5} & \multirow{2}{*}{$0.02 *$} \\
\hline & Europe & 63 & $46(21-87)$ & 40.8 & 2571.5 & & \\
\hline \multirow{2}{*}{ Age (years) } & America & 26 & $21(19-25)$ & 40.2 & 1045.5 & \multirow{2}{*}{694.5} & \multirow{2}{*}{0.26} \\
\hline & Europe & 63 & $23(20-26)$ & 47.0 & 2959.5 & & \\
\hline \multirow{2}{*}{$\begin{array}{l}\text { The number of competition } \\
\text { days in the season }\end{array}$} & America & 26 & $121(84-141)$ & 53.4 & 1387.0 & \multirow{2}{*}{602.0} & \multirow{2}{*}{0.05} \\
\hline & Europe & 63 & $99(73-120)$ & 41.6 & 2618.0 & & \\
\hline \multirow{2}{*}{ The number of days among competitions } & America & 26 & $14(11-16)$ & 62.4 & 1622.5 & \multirow{2}{*}{366.5} & \multirow{2}{*}{$0.000^{*}$} \\
\hline & Europe & 63 & $10(8-12)$ & 37.8 & 2382.5 & & \\
\hline \multirow{2}{*}{ The total number of competitions } & America & 26 & $8(6-10)$ & 36.8 & 956.0 & \multirow{2}{*}{605.0} & \multirow{2}{*}{0.052} \\
\hline & Europe & 63 & $9(7-12)$ & 48.4 & 3049.0 & & \\
\hline \multirow{2}{*}{$\begin{array}{l}\text { The number of competitions } \\
\text { in which SB was achieved }\end{array}$} & America & 26 & $4(2-5)$ & 34.4 & 894.0 & \multirow{2}{*}{543.0} & \multirow{2}{*}{$0.01 *$} \\
\hline & Europe & 63 & $5(3-6)$ & 49.4 & 3111.0 & & \\
\hline \multirow{2}{*}{$\mathrm{SB} /$ Competition } & America & 26 & $50(29.5-60)$ & 39.1 & 1016.5 & \multirow{2}{*}{665.5} & \multirow{2}{*}{0.17} \\
\hline & Europe & 63 & $55(33-71)$ & 47.4 & 2988.5 & & \\
\hline \multirow{2}{*}{ SB Performance(m) } & America & 26 & $1.85(1.84-1.89)$ & 34.9 & 906.5 & \multirow{2}{*}{555.5} & \multirow{2}{*}{$0.02 *$} \\
\hline & Europe & 63 & $1.88(1.85-1.92)$ & 49.2 & 3098.5 & & \\
\hline \multirow{2}{*}{ First performance in the season (m) } & America & 26 & $1.80(1.75-1.84)$ & 40.0 & 1039.0 & \multirow{2}{*}{688.0} & \multirow{2}{*}{0.24} \\
\hline & Europe & 63 & $1.81(1.78-1.85)$ & 47.1 & 2966.0 & & \\
\hline \multirow{2}{*}{ Last performance in the season (m) } & America & 26 & $1.80(1.78-1.83)$ & 39.1 & 1015.5 & \multirow{2}{*}{664.5} & \multirow{2}{*}{0.16} \\
\hline & Europe & 63 & $1.82(1.79-1.85)$ & 47.5 & 2989.5 & & \\
\hline \multirow{2}{*}{ Season performance average (m) } & America & 26 & $1.81(1.78-1.83)$ & 38.2 & 993.0 & 6420 & 0.11 \\
\hline & Europe & 63 & $1.82(1.79-1.86)$ & 47.8 & 3012.0 & 042.0 & 0.11 \\
\hline & America & 26 & $97(95-98)$ & 48.9 & 1271.0 & & \\
\hline First competition \% & Europe & 63 & $96(95-98)$ & 43.4 & 2734.0 & 118.0 & 0.35 \\
\hline & America & 26 & $97(95-98)$ & 44.8 & 1163.5 & & \\
\hline Last competition \% & Europe & 63 & $97(95-98)$ & 45.1 & 2841.5 & 812.5 & 0.95 \\
\hline & America & 26 & $97(96-98)$ & 49.7 & 1291.5 & & \\
\hline Aver & Europe & 63 & 97 (96-97) & 43.1 & 2713.5 & 091.5 & 0.25 \\
\hline
\end{tabular}

Note. $* \mathrm{p}<0.05$. 
Significant differences were found in favor of the European female high jumpers in groups compared in terms of the SB performances, world rankings, and the number of competitions in which SB performance was achieved. Statistically significant differences were found between the European and American high jumpers (female) in terms of the number of days between competitions $(p<0.05)$. It was observed that the American athletes had longer competition gaps (Tables 6).

Table 7. Comparison of the averages of the age and seasonal performance variables of the American and European male pole vaulters

\begin{tabular}{|c|c|c|c|c|c|c|c|}
\hline Pole Vault (Male) & Continent & $\mathbf{n}$ & Median (25-75\%) & Mean Rank & Total Rank & $\mathbf{U}$ & $\mathbf{p}$ \\
\hline \multirow{2}{*}{ World Ranking } & America & 33 & $46(21-86)$ & 44.5 & 1469.0 & \multirow{2}{*}{742.0} & \multirow{2}{*}{0.44} \\
\hline & Europe & 50 & $46(21-78)$ & 40.3 & 2017.0 & & \\
\hline \multirow{2}{*}{ Age (years) } & America & 33 & $23(20-25)$ & 37.5 & 1236.0 & \multirow{2}{*}{675.0} & \multirow{2}{*}{0.16} \\
\hline & Europe & 50 & $24.5(21-28)$ & 45.0 & 2250.0 & & \\
\hline \multirow{2}{*}{$\begin{array}{l}\text { The number of competition } \\
\text { days in the season }\end{array}$} & America & 33 & $99(93-119)$ & 39.6 & 1306.0 & \multirow{2}{*}{745.0} & \multirow{2}{*}{0.46} \\
\hline & Europe & 50 & $105(90-131)$ & 43.6 & 2180.0 & & \\
\hline \multirow{2}{*}{ The number of days among competitions } & America & 33 & $10(8-12)$ & 47.4 & 1565.5 & \multirow{2}{*}{645.5} & \multirow{2}{*}{0.09} \\
\hline & Europe & 50 & $9(8-11)$ & 38.4 & 1920.5 & & \\
\hline \multirow{2}{*}{ The total number of competitions } & America & 33 & $10(8-11)$ & 33.3 & 1098.0 & \multirow{2}{*}{537.0} & \multirow{2}{*}{$0.01 *$} \\
\hline & Europe & 50 & $12(9-15)$ & 47.8 & 2388.0 & & \\
\hline \multirow{2}{*}{$\begin{array}{l}\text { The number of competitions } \\
\text { in which SB was achieved }\end{array}$} & America & 33 & $7(3-8)$ & 41.9 & 1383.5 & \multirow{2}{*}{822.5} & \multirow{2}{*}{0.98} \\
\hline & Europe & 50 & $6(4-8)$ & 42.1 & 2102.5 & & \\
\hline \multirow{2}{*}{ SB/Competition } & America & 33 & $67(42.5-88.5)$ & 48.3 & 1593.5 & \multirow{2}{*}{617.5} & \multirow{2}{*}{0.05} \\
\hline & Europe & 50 & $55(33-71.5)$ & 37.9 & 1892.5 & & \\
\hline \multirow{2}{*}{ SB Performance (m) } & America & 33 & $5.60(5.50-5.7)$ & 39.5 & 1302.0 & \multirow{2}{*}{741.0} & \multirow{2}{*}{0.43} \\
\hline & Europe & 50 & $5.60(5.52-5.7)$ & 43.7 & 2184.0 & & \\
\hline \multirow{2}{*}{ First performance in the season (m) } & America & 33 & $5.35(5.18-5.55)$ & 39.2 & 1292.5 & \multirow{2}{*}{731.5} & \multirow{2}{*}{0.38} \\
\hline & Europe & 50 & $5.40(5.24-5.51)$ & 43.9 & 2193.5 & & \\
\hline \multirow{2}{*}{ Last performance in the season $(\mathrm{m})$} & America & 33 & $5.45(5.33-5.55)$ & 47.9 & 1580.5 & \multirow{2}{*}{630.5} & \multirow{2}{*}{0.07} \\
\hline & Europe & 50 & $5.35(5.25-5.51)$ & 38.1 & 1905.5 & & \\
\hline \multirow{2}{*}{ Season performance average $(\mathrm{m})$} & America & 33 & $5.38(5.30-5.50)$ & 41.0 & 1351.5 & & \\
\hline & Europe & 50 & $5.40(5.29-5.50)$ & 42.7 & 2134.5 & 790.5 & 0.75 \\
\hline & America & 33 & 95 (93.5-98) & 39.5 & 1302.0 & & \\
\hline First competition \% & Europe & 50 & 96 (94-98.3) & 43.7 & 2184.0 & 741.0 & 0.43 \\
\hline & America & 33 & 97 (96-99.5) & 50.6 & 1670.5 & & \\
\hline Last competition \% & Europe & 50 & $95.5(93.8-97.1)$ & 36.3 & 1815.5 & 540.5 & 0.01 \\
\hline$A_{\mathrm{u}}$ & America & 33 & 96 (95.2-97) & 43.5 & 1436.5 & 7745 & 067 \\
\hline Average \% & Europe & 50 & $96(95-97)$ & 41.0 & 2049.5 & 174.5 & 0.62 \\
\hline
\end{tabular}

Note. ${ }^{*} \mathrm{p}<0.05$. 
In the pole vault category, the total number of competitions of male (Table 7) European athletes was higher than the American athletes $(\mathrm{p}<0.05)$.

Table 8. Comparison of the averages of the age and seasonal performance variables of the American and European female pole vaulters

\begin{tabular}{|c|c|c|c|c|c|c|c|}
\hline Pole Vault (Female) & Continent & $\mathbf{n}$ & Median (25-75\%) & Mean Rank & Total Rank & $\mathbf{U}$ & $\mathbf{p}$ \\
\hline \multirow{2}{*}{ World Ranking } & America & 38 & $43(20-73)$ & 44.0 & 1673.5 & \multirow{2}{*}{932.5} & \multirow{2}{*}{0.55} \\
\hline & Europe & 53 & $56(33-69)$ & 47.4 & 2512.5 & & \\
\hline \multirow{2}{*}{ Age (years) } & America & 38 & $22(21-25)$ & 41.8 & 1586.5 & \multirow{2}{*}{845.5} & \multirow{2}{*}{0.19} \\
\hline & Europe & 53 & $24(21-27)$ & 49.1 & 2599.5 & & \\
\hline \multirow{2}{*}{$\begin{array}{l}\text { The number of competition } \\
\text { days in the season }\end{array}$} & America & 38 & $99(75-125)$ & 46.7 & 1775.0 & \multirow{2}{*}{980.0} & \multirow{2}{*}{0.83} \\
\hline & Europe & 53 & $96(80-119)$ & 45.5 & 2411.0 & & \\
\hline \multirow{2}{*}{ The number of days among competitions } & America & 38 & $11(9-15)$ & 57.3 & 2179.0 & \multirow{2}{*}{576.0} & \multirow{2}{*}{$0.000 *$} \\
\hline & Europe & 53 & $9(7-10)$ & 37.9 & 2007.0 & & \\
\hline \multirow{2}{*}{ The total number of competitions } & America & 38 & $8(7-10)$ & 33.8 & 1283.0 & \multirow{2}{*}{542.0} & \multirow{2}{*}{$0.000 *$} \\
\hline & Europe & 53 & $12(8-13)$ & 54.8 & 2903.0 & & \\
\hline \multirow{2}{*}{$\begin{array}{l}\text { The number of competitions in which } \\
\text { SB performance was achieved }\end{array}$} & America & 38 & $6(3-7)$ & 37.7 & 1431.0 & \multirow{2}{*}{690.0} & \multirow{2}{*}{$0.01 *$} \\
\hline & Europe & 53 & $7(4-11)$ & 52.0 & 2755.0 & & \\
\hline \multirow{2}{*}{ SB/Competition } & America & 38 & $58.5(40-78.5)$ & 44.2 & 1679.0 & \multirow{2}{*}{938.0} & \multirow{2}{*}{0.58} \\
\hline & Europe & 53 & $64(47-83)$ & 47.3 & 2507.0 & & \\
\hline \multirow{2}{*}{ SB Performance (m) } & America & 38 & $4.45(4.35-4.6)$ & 48.0 & 1822.5 & \multirow{2}{*}{932.5} & \multirow{2}{*}{0.55} \\
\hline & Europe & 53 & $4.42(4.35-4.51)$ & 44.6 & 2363.5 & & \\
\hline \multirow{2}{*}{ First performance in the season (m) } & America & 38 & $4.24(4.05-4.4)$ & 48.5 & 1844.0 & \multirow{2}{*}{911.0} & \multirow{2}{*}{0.44} \\
\hline & Europe & 53 & $4.20(4.00-4.43)$ & 44.2 & 2342.0 & & \\
\hline \multirow{2}{*}{ Last performance in the season $(\mathrm{m})$} & America & 38 & $4.31(4.16-4.45)$ & 49.6 & 1883.0 & \multirow{2}{*}{872.0} & \multirow{2}{*}{0.28} \\
\hline & Europe & 53 & $4.26(4.16-4.35)$ & 43.5 & 2303.0 & & \\
\hline \multirow{2}{*}{ Season performance average (m) } & America & 38 & $4.28(4.17-4.39)$ & 48.7 & 1851.0 & 0040 & 0.41 \\
\hline & Europe & 53 & $4.23(4.16-4.33)$ & 44.1 & 2335.0 & 904.0 & 0.41 \\
\hline & America & 38 & $94.5(93-98)$ & 47.2 & 1795.0 & & \\
\hline rirst competition \% 0 & Europe & 53 & $95(90-98)$ & 45.1 & 2391.0 & 900.0 & 0.10 \\
\hline $\mathbf{I}$ & America & 38 & $97(94.8-99)$ & 47.1 & 1791.0 & 0640 & \\
\hline Last Competilionit & Europe & 53 & 97 (93-99) & 45.2 & 2395.0 & 304.0 & 0.13 \\
\hline & America & 38 & $96(95-97)$ & 47.9 & 1818.5 & & \\
\hline & Europe & 53 & $96(95-97)$ & 44.7 & 2367.5 & (20.0 & 0.00 \\
\hline
\end{tabular}

Note. ${ }^{*} \mathrm{p}<0.05$. 
In the pole vault category, the total number of competitions of female European athletes was higher than the American athletes (Table 8) $(\mathrm{p}<0.05)$. There were statistically significant differences between the groups regarding the female pole vaulters category in terms of the number of days among competitions $(U=576 ; p<0.001)$ and the number of SB performances $(U=690 ; p=0.01)$ and in the male pole vaulters category in terms of the ratio of the last competition performance to the SB performance (Table 8).

\section{Discussion}

The periodization concepts and approaches of Matveyev, which were put forward in 1981, have led to the increase in the number of competitions, the quality and length of the competition period and the decrease in the phase devoted to the preparation and, thus, caused different ideas to emerge in the periodization structure (Açıkada, 2018). The present study aimed to determine the differences and similarities between the continents of America and Europe by comparing them in terms of the variables that make up the competition period.

In this study, similarities were determined between the two continents in terms of world ranking and SB performance in all jumping events except for the female events. In the female high jump event, the European athletes were better than American athletes in both world ranking and SB performance $(\mathrm{p}<0.05)$. When the all-time (top 100) performances were analyzed, it was seen that the European female high jumpers had a majority over $80 \%$ (WorldAthletics, 2009b). In light of these findings, it is possible to talk about systematic success in the female high jump event in Europe.

Another impressive finding of this study was that there were statistically significant differences between the American and European athletes in jumping events in terms of the number of days between competitions (excluding male pole vault). Similarities were detected between the continents in the male pole vault category. It can be said that the American jumpers had longer competition intervals, in other words, they exhibit longer recovery times between each competition. In the events in which similarities were determined in terms of world rankings and SB performance, differences were detected in the number of days between competitions. Accordingly, it can be said that these differences were due to the different periodization concepts between the continents. As a matter of fact, Açıkada (2018) stated that some western countries such as the United States of America have still not adopted to the concepts of periodization.

In terms of the total number of days in the competition season, significant differences were determined between the groups in favor of the American male long jumpers, male triple jumpers and female triple jumpers $(\mathrm{p}<0.05)$. Statistically significant differences were also calculated in other events. The fact that the International Sports Federation increases the number of competitions of elite athletes in one competition period affects countries and increases national competitions. As a result, athletes participate in more competitions during a competition period (Issurin, 2008).

In this study, when examining the SB performance in the competition, similarities between the American and European athletes in the male jumping events and statistically significant 
differences in the female jumping events (except female long jump) were discovered ( $\mathrm{p}<$ 0.05). It was found that SB performance was reached in the 4th-5th competition for high jump, 6th-7th for pole vault, 4th-5th competition for long jump and 3rd-4th for triple jump. Gandelsman and Smirnov (Bompa, cited in 1994 p. 190) reported that an athlete must participate in an average of 7-10 competitions before reaching peak performance. The averages determined in the present study were found to be lower than those of the literature. In high performance sports, achieving peak performance in the target competition is the main goal. As a factor affecting the athlete's SB performance, the arrangement of the competitions in the competition period (Aç1kada, 2018), the priority order of the competitions and the number of competitions before the peak performance are the topics that the training organizers emphasize.

It was emphasized that the first performances of athletes in a season, within the scope of $100 \mathrm{~m}$ event, can help to predict SB performances (Örs et al., 2019b). In the present study, it was observed that there were statistical similarities between the athletes of both continents in terms of first season performances. In other words, the importance given to the first performances in a season were the same for both European and American athletes.

When the world records in jumping events were observed it was seen that seven of the 12 records for males (seniors, U20 and U18 categories) had been achieved by European athletes, four had been achieved by American athletes and one had been achieved by an Asian athlete. While in the females, one of the 12 records had been achieved by an Australian and the remaining 11 had been achieved by European athletes. 75\% of world records jumping events for both genders had been achieved by European athletes and $17 \%$ by American athletes.

\section{Conclusions}

In conclusion, the systematic success of European athletes in jumping events was evident in many ways compared when compared to American athletes. In this study, it was determined that the training science of European athletes who participated in the competitions with shorter recovery times, generally differed significantly compared to American athletes. The superiority of European female high jumpers in terms of both SB performance and their position in the world ranking was also determined. As a result, it was thought that the differences determined between the groups may be due to the differences in the perception of periodization and different coaching educations embraced by continents.

\section{Source of Finance}

During this study, no financial or spiritual support was received neither from any pharmaceutical company that has a direct connection with the research subject, nor from a company that provides or produces medical instruments and materials which may negatively affect the evaluation process of this study.

\section{Conflict of Interest}

No conflicts of interest between the authors and/or family members of the scientific and medical committee members or members of the potential conflicts of interest, counseling, 
expertise, working conditions, shareholding and similar situations in any firm.

\section{References}

Açıkada, C. (2018). Training principles, periodization and form trainings. Training science. Sports Publishing Company.

Açıkada, C., \& Bayraktar, I. (2018). Periodization strategies on athletics. In C. Acikada (Ed.), Training Science (pp. 387-399). Sports Publishing Company.

Blumenstein, B., \& Orbach, I. (2020). Periodization of psychological preparation within the training process. International Journal of Sport and Exercise Psychology, 18(1), 13-23. https://doi.org/10.1080/1612197X.2018.1478872

Bompa, T. (1994). Theory and Methodology of Training: The Key To Athletic Performance. Hunt Publishing Company.

Bompa, T. (1999). Periodization: The theory and methodology of training. Human Kinetics Publishers.

Bradbury, D. G., Landers, G. J., Benjanuvatra, N., \& Goods, P. S. (2020). Comparison of linear and reverse linear periodized programs with equated volume and intensity for endurance running performance. The Journal of Strength and Conditioning Research, 34(5), 1345-1353. https://doi.org/10.1519/JSC.0000000000002805

Demir, M. (2008). Runnings, jumping, throws in athletics. Nobel Academic Press.

Dick, F. W. (2007). Sports Training Principles. Bloomsbury (5th ed.). Henry Kimpton. https://doi.org/10.1017/CBO9781107415324.004

Ekechukwu, R. O., \& Isiguzo, B. C. (2019). Predictive Determinants of Anxiety, Depression and Stress on Athletics Performance of Students, University of Port Harcourt, Nigeria. European Journal of Education Studies, 91-99. https://doi.org/10.5281/zenodo.2557038

IAAF. (2017). Competition Rules 2018-2019 (p. 69). Retrieved from https://www.world athletics.org/about-iaaf/documents/technical-information

Issurin, V. B. (2008). Block periodization versus traditional training theory: A review. Journal of Sports Medicine and Physical Fitness, 48(1), 65-75.

Lidor, R., Blumenstein, B., \& Tenenbaum, G. (2007). Psychological aspects of training in european basketball: Conceptualization, periodization, and planning. Sport Psychologist, 21(3), 353-367. https://doi.org/10.1123/tsp.21.3.353

Matveyev, L. (1981). Fundamentals of sports training. Progress.

Müller, H., \& Ritzdorf, W. (2009). In B. Glad (Ed.), Run! Jump! Throw! The International Association of Athletics Federations.

Örs, B. S., Cantas, F., Onarici Gungor, E., \& Simsek, D. (2019). Assessment and comparison of visiual skills among athletes. Spor ve Performans Araştırmaları Dergisi, December. 
https://doi.org/10.17155/omuspd.522342

Örs, T., Bayraktar, I., Demir, M. O., \& Örs, B. S. (2019). A competition period evaluation of elite sprinters: 100 meters event sample (pp. 830-837). II. World Congress of Sport Sciences Researches.

Raysmith, B., Jacobsson, J., Drew, M., \& Timpka, T. (2019). What Is Performance? A Scoping Review of Performance Outcomes as Study Endpoints in Athletics. Sports, 7(3), 1-14. https://doi.org/10.3390/sports7030066

WorldAthletics. (2009a). All Time Top List. Retrieved from https://www.worldathletics.org

WorldAthletics. (2009b). Season Top List. Retrieved from https://www.worldathletics.org

\section{Copyright Disclaimer}

Copyright for this article is retained by the author(s), with first publication rights granted to the journal.

This is an open-access article distributed under the terms and conditions of the Creative Commons Attribution license (http://creativecommons.org/licenses/by/3.0/). 